PROFESIONALES Y HERRAMIENTAS PARA EL DESARROLLO LOCAL Y SUS SINERGIAS TERRITORIALES. EVALUACIÓN Y PROPUESTAS DE FUTURO IX Coloquio Nacional de Desarrollo Local del GTDL-AGE 

ANTONIO MARTÍNEZ PUCHE, XAVIER AMAT MONTESINOS, ISABEL SANCHO CARBONELL y DANIEL SANCHIZ CASTAÑO (EDS.)

\section{PROFESIONALES Y HERRAMIENTAS PARA EL DESARROLLO LOCAL Y SUS SINERGIAS TERRITORIALES. EVALUACIÓN Y PROPUESTAS DE FUTURO}

IX Coloquio Nacional de Desarrollo Local del GTDL-AGE

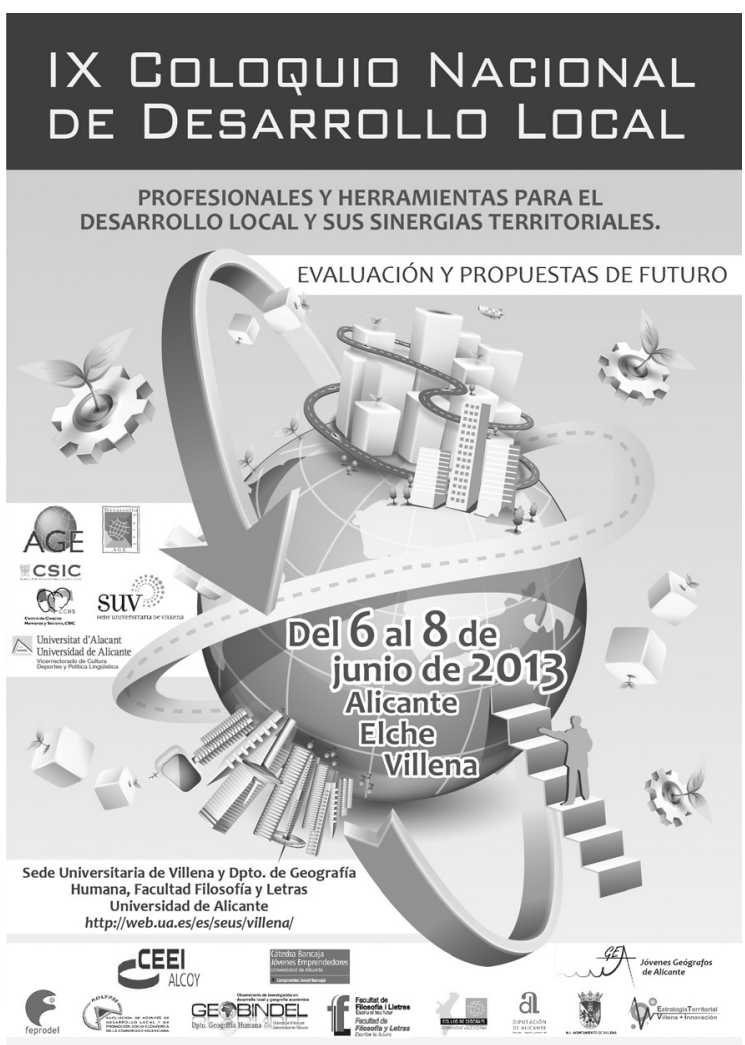


Este libro ha sido examinado y valorado por evaluadores ajenos a la Universidad de Alicante, con el fin de garantizar la calidad científica del mismo.

Publicacions de la Universitat d'Alacant

03690 Sant Vicent del Raspeig

Publicaciones@ua.es

http://publicaciones.ua.es

Telèfon: 965903480

(C) Antonio Martínez Puche, Xavier Amat Montesinos,

Isabel Sancho Carbonell y Daniel Sanchiz Castaño (eds.), 2016

(C) d'aquesta edició: Universitat d'Alacant

ISBN: 978-84-16724-00-0

Dipòsit legal: A 92-2016

Disseny de coberta: candela ink

Composició: Página Maestra (Miguel Ángel Sánchez Hernández)

Impressió i enquadernació: Guada Impresores

\section{unte \\ Unión de Editoriales
Universitarias Españolas \\ WWW.une.es
WWA}

Esta editorial es miembro de la UNE, cosa que garantiza la difusión y comercialización nacional y internacional de sus publicaciones.

Reservados todos los derechos. Cualquier forma de reproducción, distribución, comunicación pública o transformación de esta obra sólo puede ser realizada con la autorización de sus titulares, salvo excepción prevista por la ley. Diríjase a CEDRO (Centro Español de Derechos Repográficos, www.cedro.org) si necesita fotocopias o escanear algún fragmento de esta obra. 


\title{
EL SABER HACER EN LA FORMACIÓN UNIVERSITARIA EN TURISMO ANTE LA CRISIS
}

\author{
Ana de los Ángeles Torres-Rojas \\ Programa de Doctorado Dirección de Empresas Turísticas, Universidad de Huelva \\ anaangeles.torres@gmail.com \\ $F^{c o}$ Javier García-Delgado \\ Instituto de Desarrollo Local, Universidad de Huelva \\ fcogarci@uhu.es
}

\section{RESUMEN}

La crisis económica ha llevado a replantear las actividades económicas, la productividad, la innovación... En este contexto, y en el de la reforma del sistema universitario español en el marco del EEES, la implantación de los grados y el aprendizaje por competencias se presentan como una oportunidad para la mejora del sector turístico concibiéndose la formación como una baza para conseguir el ansiado desarrollo local.

El objetivo de la presente comunicación es analizar las competencias en los estudios de Grado en Turismo vinculadas con el saber hacer, la innovación y los cambios del sector como elementos dinamizadores de la empresa turística.

Palabras-clave: Formación por competencias, saber hacer, grados universitarios, desarrollo local.

\section{THE KNOW-HOW IN THE TOURISTC STUDIES FACING THE CRISIS}

\section{Abstract}

Due to the current economic crisis there has been a reconsideration of the economic activities, productivity, innovation... In this context, and as regards the reform in the Spanish University according to the ESSE, the establishment of the new degrees and learning by competences are conceived as an opportunity to improve touristic sector and a tool to achieve the local development. 
The purpose of this communication is to analyze the competences in the touristic Degrees in Spain which are linked with the know-how, the innovation and the new situation of the sector as an impulse to the touristic business.

Keywords: learning by competences, know-how, degrees, local development.

\section{INTRODUCCIÓN: LA FORMACIÓN Y EL DESARROLLO LOCAL}

En el contexto del desarrollo local (como proceso) debe entenderse el capital humano como un pilar básico, constituyéndose la formación de la población en un instrumento fundamental para su consecución.

Al contrario de lo que se ha venido pensando por parte de la sociedad (factor psico-sociológico) se ha demostrado que el desarrollo del capital humano no se vincula directamente con la posesión de títulos (parámetro cuantitativo, que no necesariamente debe equipararse con desarrollo, como puede demostrarse con las ciudades-dormitorio o la emigración internacional de titulados) sino con el desarrollo de "competencias profesionales", entendidas como conocimientos y capacidades que permiten el ejercicio de la actividad profesional conforme a las exigencias de la producción y el empleo, desde una perspectiva cualitativa (no sólo qué saben los individuos, sino también qué son capaces de hacer).

En 1988, R. Lucas introdujo la variable "capital humano" en el modelo neoclásico de crecimiento, considerando como tal el nivel general de competencias (HERRERA, 2011; 3), sobre esta base, establecía que (LUCAS, 1988; tomado de HERRERA, 2011; 3):

- Una persona más formada es más productiva e incrementa la productividad de todos los factores de producción.

- Un bajo nivel de capital humano genera que el capital físico sea menos productivo y si ambos son menores que en otras economías, su nivel de ingresos también tenderá a ser menor.

- No existe flujo de capitales de las regiones ricas a las pobres si el nivel de capital humano de éstas es muy bajo respecto al de las primeras.

- Una vez acumulada una cierta masa crítica de capital humano, ésta genera fuertes externalidades y se constituye en el principal factor de atracción o expulsión de capital físico y humano de un territorio determinado.

Desde esta perspectiva, debe concebirse la formación del mercado de trabajo como eje central de la empresa, que lo identifica como un factor de localización de actividades a veces por encima de otros (como indica CASANOVA, 2004; 61). La salida de la crisis, desde el desarrollo local, basado en los recursos endógenos, pasa por la formación de los recursos humanos (RRHH) para su inserción en el tejido productivo y el planteamiento de alternativas ante la crisis (basadas en el emprendimiento, la innovación, la flexibilidad, etc.), creando nuevas alternativas o respondiendo a las nuevas demandas del mercado laboral. 
En el contexto de crisis, de pérdida de derechos laborales o recorte de prestaciones, la formación es clave para el incremento de la competitividad y la protección del empleo como expresión de una sociedad equitativa.

\section{UNIVERSIDAD, DESARROLLO LOCAL Y FORMACIÓN}

Desde hace décadas se cuestiona el papel tradicional de la Universidad como mera depositaria del conocimiento y transmisora a través de circuitos cerrados. Ello ha motivado que los tres ejes clásicos en los que se apoyaba: docencia, investigación y extensión, tomen un nuevo camino en la sociedad actual, al buscar fórmulas para aportar a la misma y a los territorios a los que se vinculan las actividades.

En el caso español, la proliferación de Universidades públicas (o centros de educación superior en el caso de las Comunidades Autónomas que han optado por Universidades con Campus en las diferentes provincias) en los últimos cuatro decenios ha sido clave para la mejora del nivel medio de la formación de la población, por la cercanía de instituciones universitarias al conjunto del territorio del Estado ${ }^{1}$. Por otra parte, las transferencias en investigación al mundo de la empresa se han ido multiplicando, estando grupos de investigación de Universidades públicas a la cabeza de diferentes campos científicos (patentes, investigaciones punteras, laboratorios de referencia...). La extensión universitaria, por su parte, ha propiciado la difusión territorial de la cultura a través de cursos y eventos diversos, que en muchos casos han superado lo estrictamente local para convertirse en provinciales, regionales, nacionales e, incluso, internacionales.

Sin embargo, en el eje educativo, la Universidad ha estado centrada en el saber enciclopédico, la transmisión de conocimientos básicos o avanzados del profesorado al alumnado, a través de clases magistrales, evaluando conocimientos sin más. Tanto ha sido así, que, en 1997, F. Alburquerque planteaba la necesidad de cuestionarse el papel desempeñado por la universidad en cuanto a la adecuación de sus planes de estudios, su flexibilidad y carácter pluridisciplinar y, sobre todo, su alcance en cuanto a la aplicación de conocimientos orientados al desarrollo del territorio concreto (en el que radica).

En el marco legal actual, Constitución Española, LOU (6/2001, de 21 de diciembre; reformada por la Ley Orgánica 4/2007), así como en buena parte de los Estatutos de las Universidades españolas (adaptados a la LOU y las normativas autonómicas de referencia) se establecen las funciones de la Universidad (LOU, 6/2011; $\operatorname{Art}^{\circ} 1$ ):

1 En el caso de la Universidad de Huelva, el Rector (2005-2013), Francisco J. Martínez López en los discursos de Graduación de las diferentes promociones recuerda: "gran parte de quienes se gradúan hoy son la primera generación de universitarios de sus familias". 
1. La Universidad realiza el servicio público de la educación superior mediante la investigación, la docencia y el estudio.

2. Son funciones de la Universidad al servicio de la sociedad:

a) La creación, desarrollo, transmisión y crítica de la ciencia, de la técnica y la cultura.

b) La preparación para el ejercicio de actividades profesionales que exijan la aplicación de conocimientos y métodos científicos y para la creación artística.

c) La difusión, la valorización y la transferencia al servicio de la cultura, de la calidad de vida, y del desarrollo económico.

d) La difusión del conocimiento y la cultura a través de la extensión universitaria y la formación a lo largo de toda la vida.

De esta forma, la Universidad tiene la responsabilidad de promover encuentros entre los actores sociales, propiciando en ellos capacidades y potencialidades necesarias para construir identidades socio-culturales para la transformación y el cambio constante (GUTIÉRREZ, 2006; 140), la transmisión de valores y la transferencia de conocimientos (GUTIÉRREZ, 2006; 141) y, por primera vez, preparar para el ejercicio de actividades profesionales a los estudiantes, lo que entronca directamente con la vinculación de la Universidad con la realidad productiva territorial (ALBURQUERQUE, 1997; HERRERA, 2011).

Desde hace unos años, es posible establecer (RODRÍGUEZ Y VILLENEUVE, 2001; 296):

- Una tendencia general hacia la territorialización de la Universidad, enfocada hacia la sociedad que le rodea.

- Un compromiso local de la Universidad, a través de pactos con agentes sociales y económicos del entorno territorial.

- La contribución esencial de la Universidad al desarrollo territorial mediante el incremento del capital social.

Debemos entender que las Universidades (y centros de investigación) se encuentran entre los actores principales del desarrollo local (CIENFUEGOS Y AGUILAR, 2011; 220), junto con empresas y emprendedores, asociaciones empresariales y profesionales, instancias gubernativas escalares, administración pública (desconcentrada y descentralizada) y organizaciones no gubernamentales. Pero es preciso no olvidar que, "Para que la Universidad contribuya al crecimiento económico es necesario abordar cuestiones de calidad y de oferta educativa, de articulación con el mercado laboral y productivo regional como así también cuestiones relacionadas con las características propias de los territorios" (HERRERA, 2011; 4). Así, si toda iniciativa de desarrollo local busca potenciar las capacidades endógenas de la región y mejorar la calidad de vida de sus pobladores (CASANOVA, 2004; 67) la Universidad debe generar formación (técnica, profesional, continua) para el empleo, contribuir al desarrollo de redes y dinamizar territorios. 
En este sentido, la Declaración de Bolonia de junio de 1999 con vistas a la conformación del Espacio Europeo de la Educación Superior (EEES) pretendía crear un sistema de referencia para la transferencia de créditos (ECTS) que permitiese la movilidad de estudiantes y profesorado, así como converger hacia un modelo universitario menos centrado en la transmisión de conocimientos y más abierto a la formación de individuos (valores o competencias transversales) y de profesionales (competencias específicas). Sin embargo, desde nuestra perspectiva, ello no quiere decir que la Universidad tenga una visión teleológica o utilitaria (a modo de "Formación Profesional"), sino que contribuya a la construcción de escenarios de desarrollo con la formación de capital humano, conforme a las necesidades territoriales, sociales (fortalecimiento de capital social, generación de valores) y empresariales (relación positiva/complementaria con el sector productivo) (HERRERA, 2011; 1). Así pues, "el desafío consiste en generar una oferta educativa y de formación que permita, por un lado, adecuar los contenidos curriculares a las demandas de cada territorio de referencia y, por otro, anticipar las demandas futuras de cualificación que las instituciones públicas y privadas van a presentar" (MADOERY, 2003; 2).

Su plasmación en la Universidad española se producirá con el establecimiento de perfiles profesionales para las nuevas titulaciones de Grado, desarrolladas en España a partir del RD 1393/2007 (de 29 de octubre, por el que se establece la ordenación de las enseñanzas universitarias oficiales), y la implementación de nuevos marcos de referencia para la enseñanza y la evaluación formativa (basada en el desarrollo de competencias por parte del alumnado). En un paso más allá, la Universidad debe asumir un papel de formación integral, dando marcado carácter profesional a algunas enseñanzas, como las de posgrado, las reguladas (Derecho, Medicina, etc.) y aquellas con un claro carácter sectorial, que también debieran ser reguladas, como es el caso de Turismo.

Ahora se trata, en el escenario de crisis, de "provocar el desarrollo", desde una visión innovadora de la enseñanza universitaria, demostrando la capacidad dinámica de superar situaciones adversas y la capacidad estratégica del saber hacer frente al saber disciplinar.

\section{LOCALIZACIÓN DE LAS ENSEÑANZAS UNIVERSITARIAS EN TURISMO}

La Convergencia Europea y el desarrollo del Real Decreto 1393/2007 han supuesto el desarrollo de los denominados Grados. De esta forma, todas las carreras universitarias han pasado a tener una duración de cuatro cursos académicos y 240 créditos ECTS. Ello significa el paso de las Diplomaturas de tres cursos, como era el caso de los estudios universitarios de Turismo, a Grados de cuatro cursos.

En el curso 2012-2013, la oferta académica de Grados en Turismo en España es de 66 (con diferentes denominaciones) a los que se suman los Dobles 
Grados, en los que Turismo aparece, fundamentalmente, como un complemento a la formación en Administración y Dirección de Empresas (estos Dobles Grados son excluidos del análisis posterior).

De los 66 Grados un total de $37(56,07 \%)$ se imparten en universidades públicas, 14 en universidades privadas $(21,21 \%)$ y los 15 restantes $(22,72 \%)$ en centros adscritos a universidades públicas. En algunos casos, las universidades públicas ofertan un Grado en Turismo y centros adscritos a las mismas también (destaca la Universitat de Girona que a su oferta propia suma cinco centros adscritos con sede en la provincia de Barcelona). En lo que respecta a los planes de estudios, en todos los casos han sido verificados por la ANECA o agencias autonómicas responsables. La oferta académica presencial supone un total de 60 Grados y la no presencial 6 (tres de ellos en universidades públicas: UNED, Universidad de Las Palmas de Gran Canaria, Universidad Rey Juan Carlos; y otros tres en universidades privadas: UDIMA, Universidad San Antonio de Murcia, y Universitat Oberta de Catalunya).

Territorialmente, los grados se distribuyen por la geografía estatal, en un total de 32 provincias, más las islas de Mallorca e Ibiza en Baleares y Lanzarote, Gran Canaria y Tenerife en Canarias. Sin embargo, en un estudio en detalle llama la atención la inexistencia de esta titulación en alguna Comunidad

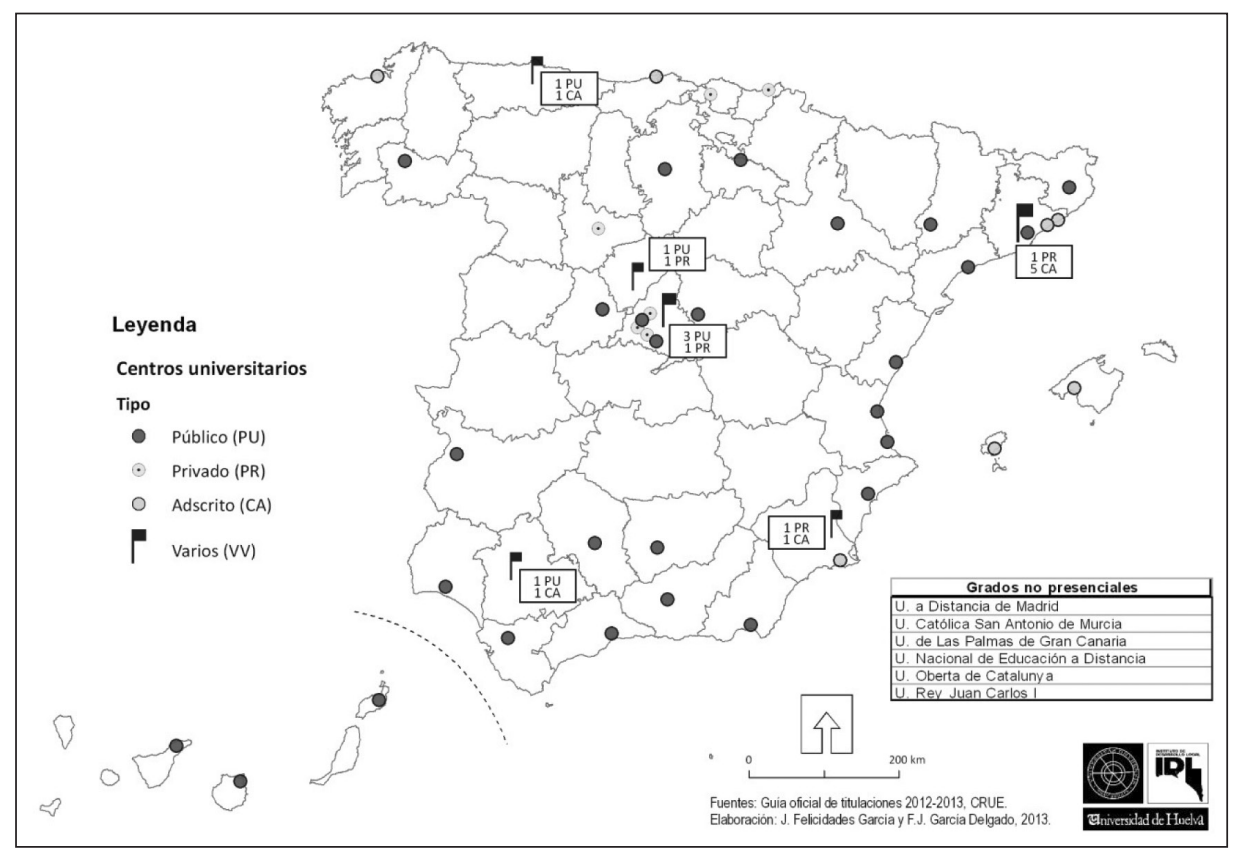

Mapa 1. Localización de los Grados en Turismo en España.

Fuente: Elaboración propia. 
Autónoma (Comunidad Foral de Navarra). En el caso de Andalucía, Cataluña y Valencia el conjunto de las provincias tienen estudios de Grado en Turismo. Destacan especialmente las provincias de Madrid y Barcelona, por el número de universidades y por la propia demanda (de todo tipo: universidades públicas, privadas y centros adscritos). Llamativa es la ausencia de estudios de Grado en Turismo en Santiago de Compostela y Salamanca (la USAL imparte estos estudios en Ávila). Al mismo tiempo, provincias con escasa oferta y demanda turística aparecen con la titulación, como es el caso de Badajoz, Segovia, Ávila u Ourense. Por otra parte, en el caso de la Comunidad Autónoma Vasca sólo hay oferta en la Universidad de Deusto, privada. En la Comunidad de Murcia existen tres Grados en Turismo, en dos centros adscritos (a la Universidad de Murcia y a la de Cartagena) y una universidad privada (San Antonio de Murcia, que oferta también una titulación no presencial).

\section{LAS NUEVAS NECESIDADES DE FORMACIÓN EN EL SECTOR TURÍSTICO}

La importancia del Turismo como actividad económica en el mundo y en España es incuestionable, no sólo por el impacto que genera de forma directa sino por la forma en que influye en otros sectores gracias a su transversalidad.

No obstante, generalmente se le ha concedido más importancia a la experiencia que a la formación. Mientras que en otros países la formación turística crecía y evolucionaba a la par que lo hacía la propia actividad, hasta 1963 no existiría en España un marco legal que regulara unos estudios en Turismo. Es por ello que a menudo el aprendizaje se llevaba a cabo dentro de las mismas empresas y se contaba con personal poco cualificado y/o proveniente de otras áreas relacionadas con el Turismo.

En la mayoría de los casos, la formación de los directivos venía dada por la propia experiencia. Sólo en casos excepcionales, se les formaba en las prestigiosas escuelas europeas. Respecto a los mandos intermedios, se trataba normalmente de trabajadores ascendidos gracias al desempeño excelente en un puesto inferior. Por último, el personal base procedía de dos situaciones: la de primer empleo (como aprendices generalmente) y la de proveniencia de sectores tradicionales como la agricultura y la construcción. A lo anterior debemos añadir que las profesiones asociadas al Turismo han adolecido de falta de valoración y prestigio, se relacionaban con niveles bajos dentro de las organizaciones y menos valorados que los de similar categoría en otros sectores. En general, la turística ha sido una profesión poco atractiva tanto para los empleados como para la comunidad educativa.

Actualmente los estudios en Turismo afrontan un proceso de cambio: en la formación no universitaria, porque nacen multitud de nuevos títulos (como fruto de la aparición de nuevas figuras profesionales y para cubrir otras ya existentes para las que no había título específico); en la universitaria, por el 
paso del modelo de enseñanza tradicional (donde priman los conocimientos teóricos, impartidos por el docente) a otro basado en el aprendizaje por Competencias (aquí el objetivo es dotar al alumno de instrumentos para que desarrolle actividades cognitivas, procedimentales y actitudinales que les conviertan en profesionales completos). De esta idea parten los nuevos Grados universitarios. "La universidad no es una Institución aislada, sino una entidad enclavada en un entorno económico, político y social cuya vinculación más orgánica se da a través de la formación de profesionales con los conocimientos y habilidades necesarias para el óptimo desempeño laboral. De acuerdo a las nuevas exigencias de las actividades económicas de la sociedad la necesidad de pertinencia ha adquirido nuevas dimensiones y mayor urgencia" (ALBORNOZ, 1999; tomado de GÓMEZ, 2009; 50). Precisamente, la formación por Competencias tiene su origen en las aportaciones del mundo del trabajo, ya que se ha observado en diversos profesionales que no transfieren al ejercicio profesional los conocimientos adquiridos, poseen la formación, pero no saben cómo aplicarla en situaciones concretas de trabajo (MÁRQUEZ Y DÍAZ, 2005).

La sociedad en general ha experimentado una serie de cambios en los últimos años que han provocado una variación en la concepción que se tenía de la actividad turística. POON, en 1993, resumía estos cambios en el sector turístico: nuevos consumidores, nuevas tecnologías, nuevas formas de producción, nuevos estilos de gestión y nuevas condiciones del entorno.

El cliente ha pasado de ser un mero consumidor de paquetes turísticos a buscar una experiencia única que satisfaga unas expectativas cada vez más altas. El mayor nivel de formación de los turistas, el volumen de información a su alcance y la diversificación de la oferta le confieren un mayor poder de decisión de compra.

Internet ha supuesto una auténtica revolución para esta actividad, tanto para la compra como para la comercialización. Estamos ante una desintermediación del sector: las empresas pueden llegar directamente al consumidor final para ofrecer sus productos. Esto exige llevar a cabo políticas comerciales mucho más dinámicas para adaptarse a un mercado en constante cambio.

En el momento actual no podemos olvidar la crisis por la que atravesamos. Si bien una crisis no debe entenderse siempre como algo negativo: también impulsa el cambio y algunas (las provocadas por conflictos bélicos o desastres naturales) conllevan una redistribución de los viajeros a otras zonas que se ven favorecidas. Lamentablemente, nos enfrentamos a una crisis económica y financiera que ha hecho retraerse el sector turístico a nivel mundial. El sector tiene que hacer frente a la misma con cambios que le ayuden a ser más competitivo: ajustes para conseguir mejores precios, innovación para lograr la diferenciación y políticas comerciales más agresivas.

Todos estos cambios influyen en la gestión de las organizaciones, que deben adaptarse a los que acaecen a su alrededor. Además, aspectos como 
la calidad, la reputación o la gestión de los RRHH se consideran ahora clave dentro de la estrategia de las empresas, que tradicionalmente han estado enfocadas a aspectos puramente operacionales, donde los resultados son fácilmente cuantificables.

Por su naturaleza, podemos afirmar que el Turismo es un sector en constante cambio, uno de los más sensibles a los efectos de la globalización. En este entorno cada vez más competitivo, globalizado y complejo, está obligado a adaptarse e incluso reinventarse para no perder su posición (en algunos casos con especial urgencia, dada la madurez de ciertos destinos). Se precisa mayor capacidad de respuesta y flexibilidad ante el cambio, creatividad para hacer frente a los competidores y profesionales capaces de satisfacer a un cliente cada vez más experimentado y exigente.

En el escenario actual, los profesionales del sector se han convertido en una pieza clave para lograr la ansiada diferenciación que augura el éxito, desde quienes gestionan las empresas, hasta el personal base, que es el nexo entre empresa y cliente. Es el toque humano el que hace que un producto turístico roce la excelencia.

Por ende, todos los cambios que experimente o quiera emprender el sector se verán reflejados en las competencias requeridas a sus profesionales. El futuro de las empresas turísticas depende de la preparación y cualificación de sus RRHH, en cualesquiera niveles de la organización (URIEL Y MONFORT, 2001). Se demandan profesionales capaces de evolucionar a la misma velocidad que lo hace el mercado o, incluso, de anticiparse a estos cambios. Así, la gestión de los recursos intangibles se convierte en pieza clave de la estrategia de la empresa ya que en ellos está la clave para la diferenciación frente a los nuevos destinos: ofrecer al turista una experiencia única.

Como ya hemos mencionado, la universidad no es un ente aislado, sino que se nutre (y así ha de ser) de lo que sucede en su entorno. Por tanto, de forma paralela a los cambios globales y en la concepción de la actividad turística y a la gestión de las empresas para ser más competitivas, debe evolucionar la formación, para que la comunidad educativa pueda procurar al mercado los profesionales que demanda y asegurar además la inserción laboral de sus titulados.

Si bien en el modelo tradicional, el profesor era el sujeto activo y se limitaba a transmitir conocimientos al alumnado y a evaluar si éste los había adquirido (con independencia de que fuera capaz de emplearlos en su futura carrera profesional o no), en la formación por Competencias el objeto no es tanto esa formación en conocimientos sino dotar al alumno de los instrumentos necesarios para que desarrolle habilidades cognitivas, procedimentales y actitudinales. Estas habilidades se materializan en Competencias. El equilibrio en el desarrollo de las Competencias garantiza la formación de profesionales completos y no de simples seres intelectuales o ejecutores. 
5. ANÁlisis de COMPETENCIAS INNOVAdORAS EN LOS GRADOS EN TURISMO DE LAS UNIVERSIDADES ESPAÑOLAS

En 2004, la ANECA elaboró el Libro Blanco de los estudios en Turismo. Se planteó como un análisis de los títulos existentes, una reflexión sobre las necesidades formativas del sector y una adaptación de los estudios universitarios en Turismo a las exigencias del nuevo marco del EEES.

Una de las prioridades de este estudio fue determinar las figuras profesionales que tendrían cabida dentro del nuevo título de Grado en Turismo, tanto de forma horizontal (dado el carácter transdisciplinar de la actividad turística) como vertical (en función de los diferentes niveles de responsabilidad dentro de las organizaciones) para, posteriormente, diseñar la estructura del plan en función de estos perfiles a alcanzar.

Por otro lado, siguiendo las directrices marcadas en el EEES sobre la formación por competencias, se elabora un listado de las competencias que los alumnos deberían haber adquirido al finalizar sus estudios universitarios en Turismo y que se dividen en actitudinales (ser), cognitivas (saber) y procedimentales o instrumentales (saber hacer).

De un total de 32 competencias específicas y 3 transversales (que se entienden implícitas en las anteriores), cabe destacar que las mejor valoradas por el profesional sector son (ANECA, 2005):

- Tener una marcada orientación de servicio al cliente (CE6)

- Trabajar en inglés como lengua extranjera (CE15)

- Definir objetivos, estrategias y políticas comerciales (CE11)

- Utilizar y analizar las Tecnologías de la Información y las Comunicaciones (TIC) en los distintos ámbitos del sector turístico (CE24)

- Manejar las técnicas de comunicación (CE13)

De aquí extraemos dos datos relevantes: las mejor valoradas son las competencias de carácter instrumental, en correspondencia con la nueva forma de concebir la formación; estas competencias dan respuesta a los cambios generales que comentábamos anteriormente y que han provocado un cambio en la actividad turística.

Para cerrar el ciclo (cambios globales que provocan cambios en el sector turístico, que a su vez repercuten en el perfil profesional de los RRHH y, por tanto, en los estudios que forman para estos perfiles), tendríamos que ver cómo los planes de estudio se hacen eco de estas competencias. La estructura de estos planes varía en función de las Comunidades Autónomas, entre las que se registran similitudes ${ }^{2}$, y todos tienen como base el mencionado Libro Blanco de la ANECA (2005) y el marco de referencia MECES (Marco Español de Cualificaciones para la Educación Superior). En el análisis se ha procedido

2 Cada Comunidad Autónoma creó comisiones de Título y de Rama para que estableciesen la Formación Básica y Obligatoria en los diferentes Grados. 
Tabla 1. Plasmación de las Competencias del Libro Blanco en los planes de estudios de Grados en Universidades públicas

\begin{tabular}{|c|c|c|c|c|c|}
\hline \multirow[b]{2}{*}{ Universidad } & \multicolumn{5}{|c|}{ Competencias del Libro Blanco } \\
\hline & $\begin{array}{l}\text { Tener una marcada } \\
\text { orientación de } \\
\text { servicio al cliente } \\
\text { (CE6) }\end{array}$ & $\begin{array}{l}\text { Trabajar en inglés } \\
\text { como lengua } \\
\text { extranjera (CE15) }\end{array}$ & $\begin{array}{l}\text { Definir objetivos, } \\
\text { estrategias y } \\
\text { políticas comerciales } \\
\text { (CE11) }\end{array}$ & $\begin{array}{l}\text { Utilizar y analizar } \\
\text { las TIC en los } \\
\text { distintos ámbitos } \\
\text { del sector turístico } \\
\text { (CE24) }\end{array}$ & $\begin{array}{l}\text { Manejar las técnicas } \\
\text { de comunicación } \\
\text { (CE13) }\end{array}$ \\
\hline UA & CE6 & CE15 & CE11 & CE24 & CE13 \\
\hline UAB & No aparece & No aparece & No aparece & CT (24) & No aparece \\
\hline UAH & CE6 & CE15 & CE11 & CE22 & CE13 \\
\hline UAL & GET10 & IMP01 & GET05 & IGT01 & IMT04 (1) \\
\hline UAM & CE6 & CE15 & CE11 & CE24 & CE13 \\
\hline UBU & CE4 & (25) & No aparece (26) & CE17 & CE10 \\
\hline UC3M & No aparece & CE6 (28) & CE4 (27) & No aparece & No aparece \\
\hline UCA & CE6 & CE15 (2) & CE11 & CE24 & CE13 \\
\hline UCO & (3) & CE25 (4) & CE18 (5) & CU2 (6) & No aparece \\
\hline UDG & CE19 & CE4 (7) & CE28 & CE34 (8) & CE14 (9) \\
\hline UDL & & CEst2/CE 8 (29) & CE6 (31) & Cest3 (30) & No aparece \\
\hline UGR & CE3 (10) & CE3 (10) & CE1 (11) & CT5 (12) & CE1 (11) \\
\hline UHU & CT14 & CE46 (13) & CE30 & СT6 & CE41 (14) \\
\hline UIB & (19) & CE1 (15) / CE8 (17) & CE13 (18) & CE7 (16) / CE8 (17) & (19) \\
\hline UJAEN & CE6 & CE15 & CE11 & CE24 & CE13 \\
\hline UJI & CE6 & CE15 & CE11 & CE24 & CE13 \\
\hline ULL & CE6 & CE15 & CE11 & CE24 & CE13 \\
\hline ULPGC & CE5 & CE15(20) & CE11 & CE22 & CE13 \\
\hline UMA & CE6 & CE15 & CE11 & CE24 & CE13 (1) \\
\hline UNED & CE2.6 & CE2.5 (32) & CE1.6 (34) & CE3.1 (33) & No aparece \\
\hline UNEX & CE27 & CE28 & CE17 & CE3 & CE24 (1) \\
\hline UNIOVI & CE6 & CE15 & CE11 & CE24 & CE13 \\
\hline UNIRIOJA & CE6 (35) & CE15 (36) & CE11 (36) & CE23 (36) & CE13 (36) \\
\hline UNIZAR & CE5 & CE18 (21) & CE11 & CE25 & CE15 \\
\hline UPV & No aparece & CG4 (37) & (38) & CG5 & (38) \\
\hline URJC & CE30 & CE31 & CE20 & CE3 & CE27 \\
\hline URV & CA10 (39) & $\mathrm{CCl}(40)$ & CE-8 (42) & CC2 (41) & CE-8 (42) \\
\hline US & No aparece & CE30 & CE16 & CE22 & CE33 (1) \\
\hline USAL & CE6 & CE15 & CE11 & CE24 & CE13 \\
\hline
\end{tabular}




\begin{tabular}{|l|c|c|c|c|c|}
\hline \multirow{4}{*}{ Universidad } & \multicolumn{5}{|c|}{ Competencias del Libro Blanco } \\
\cline { 2 - 6 } & $\begin{array}{c}\text { Tener una marcada } \\
\text { orientación de } \\
\text { servicio al cliente } \\
\text { (CE6) }\end{array}$ & $\begin{array}{c}\text { Trabajar en inglés } \\
\text { como lengua } \\
\text { extranjera (CE15) }\end{array}$ & $\begin{array}{c}\text { Definir objetivos, } \\
\text { estrategias y } \\
\text { políticas comerciales } \\
\text { (CE11) }\end{array}$ & $\begin{array}{c}\text { Utilizar y analizar } \\
\text { las TIC en los } \\
\text { distintos ámbitos } \\
\text { del sector turístico } \\
\text { (CE24) }\end{array}$ & $\begin{array}{c}\text { Manejar las técnicas } \\
\text { de comunicación } \\
\text { (CE13) }\end{array}$ \\
\hline UV & CP6 & CP15 & CP11 & CP24 & CP13 \\
\hline UVA & CE29 & CE30 & CE19 & CE32 & CE26 \\
\hline UVIG0 & C2.6 & CE2.5 (22) & C1.6 (23) & C3.1 & C1.6 (23) \\
\hline
\end{tabular}

CE: COMPETENCIA ESPECÍFICA. CP: COMPETENCIA PROFESIONAL.

(1) Aplicada sólo al idioma extranjero. (2) Modificada parcialmente: Comunicarse de forma oral y escrita en inglés como lengua extranjera. (3) Parcialmente recogida en la Competencia Básica 5. (4) Modificada parcialmente: Trabajar en inglés como lengua extranjera. (5) Modificado parcialmente: Definir objetivos, estrategias y políticas comerciales en el ámbito del turismo. (6) Competencia de la Universidad: Conocer y perfeccionar el nivel de usuario en el ámbito de las TIC. (7) Parcialmente modificada como: Utilizar la lengua inglesa y otras lenguas extranjeras. (8) Modificado parcialmente como: Seleccionar y utilizar las tecnologías de la información y la comunicación más adecuadas a cada situación. (9) Modificada parcialmente: Ser hábil en la comunicación utilizando técnicas/recursos diversos. (10) Recogidas en una competencia más amplia: Capacidad para trabajar en una organización turística adoptando una orientación hacia el cliente y estableciendo relaciones satisfactorias basadas en la generación de conocimiento y el manejo de técnicas de comunicación e idiomas. (11) Contenidas en una competencia más amplia: Capacidad para gestionar un destino turístico o una organización (empresas e instituciones), así como de llevar a cabo la promoción y comercialización, y la planificación turística, tanto en el conjunto del destino turístico o de la organización, como en cualquiera de sus áreas funcionales. (12) Recogida en parte como competencia transversal: Conocimientos de informática relativos al ámbito de estudio. (13) Recogida en una competencia más amplia: Trabajar en lengua extranjera. (14) Recogida en una competencia más amplia: Manejar técnicas de comunicación en lengua nativa y extranjera. (15) Recogida en la competencia más genérica: Adquirir un conocimiento de la lengua inglesa para, en un nivel medio, comprender, hablar y escribir en dicho idioma de forma que garantice la comprensión, expresión y aplicación de la terminología específica relacionada con el turismo en los principales campos profesionales que estén relacionados. (16) Recogida en parte en una competencia más amplia: Aprender a recopilar, procesar, analizar e interpretar la información y a hacer frente a cuestiones turísticas a través del despliegue de habilidades específicas para el trabajador en las distintas ramas turísticas, incluida la del uso apropiado de tecnologías de información y comunicación (TIC). (17) Ambas son abordadas parcialmente en otra más amplia: Demostrar conocimiento y comprensión en diferentes lenguas, distintas a la materna, así como lograr el dominio de la tecnología asociada al mercado turístico general, con la adquisición y análisis de datos, los datos estadísticos, las técnicas de reservas on line, de análisis cualitativos y de aplicaciones informáticas. (18) Comprendida en otra más amplia: Demostrar el dominio de una amplia gama de estrategias analíticas y de observación desarrolladas a partir de los métodos habituales de investigación aportados por las distintas ramas cientíicas a lo largo de su etapa académica y la concreción en el trabajo correspondiente. (19) Integradas en asignaturas específicas: Habilidades Directivas, Gestión de la Calidad y Atención al Cliente. (20) Parcialmente modificada, al incorporar el uso de una segunda lengua: Dominio del inglés y perfeccionamiento e intensificación, como mínimo, de una segunda lengua extranjera. (21) Integrada en otra más amplia: Comunicarse de forma oral y escrita en lengua inglesa en el entorno de trabajo de la actividad turística. (22) Concretada: Desempeñar actividades profesionales en inglés dentro del sector turístico. (23) Recogida en otra más amplia: Los fundamentos del marketing turístico, así como sus objetivos, estrategias y políticas comerciales. (24) Integrada en una competencia transversal: Adaptarse a los cambios tecnológicos y utilizar todas las herramientas de comunicación al alcance. (25) Integrada en Comunicarse en inglés y en otras lenguas extranjeras. (26) Plan con marcado carácter planificador y de aprovechamiento del patrimonio. (27) Recogida en Diseñar productos turísticos y definir objetivos, estrategias y políticas comerciales. (28) Comprendida en Comunicarse de forma oral y escrita en lenguas extrajeras. (29) Incorporada a la competencia estratégica Coneixement i domini de l'anglès com a llengua estrangera y específica Coneixement i domini de llengües estrangeres. (30) Incorporada a la competencia estratégica Domini de les TIC. (31) Incorporada como Capacitat de dissenyar productes turístics i definir objectius, estratègies i polítiques comercials. (32) Recogida en Desempeñar actividades profesionales en inglés dentro del sector turístico. (33) Recogida en Habilidades en el manejo de las TIC para la búsqueda y aprovechamiento de la información. (34) Recogida en Los fundamentos del marketing turístico así como objetivos, estrategias y políticas comerciales. (35) Redactada como Comprensión de la relevancia de la orientación de servicio al cliente para el sector turístico. (36) Aparecen como "Capacidad de...". (37) Competencia Genérica redactada como Comunicarse de forma oral y escrita en inglés y en una segunda lengua extranjera. (38) Integrada en una asignatura: Planificación comercial de empresas turísticas y estrategias de comunicación para destinos turísticos. (39) Se amplía como Tener una marcada orientación de servicio al cliente y una motivación por la calidad del servicio. (40) Formulada como Dominar en un nivel intermedio una lengua extranjera, preferentemente el inglés. (41) Redactada como Utilizar de manera avanzada las tecnologías de la información y la comunicación. (42) Reformuladas como Definir objetivos, estrategias y políticas comerciales y técnicas de comunicación.

Fuente: Memorias de Grado de los diferentes títulos. Elaboración Torres-Rojas y García-Delgado, 2013. 
a seleccionar seis Grados en Universidades públicas: Alicante, Vigo, Girona, Rey Juan Carlos, Zaragoza y Huelva, que representan a las tres Comunidades Autónomas en las que se imparte el Grado en Turismo en todas sus provincias (Comunidad Valenciana, Cataluña y Andalucía), además de las Comunidades de Galicia, Comunidad de Madrid y Aragón.

La memoria del título de Grado en Turismo de la Universidad de Alicante incluye, además de las competencias establecidas por el Libro Blanco (específicas), una serie de competencias generales que se entienden implícitas en las otras y que hacen referencia a los idiomas, las TIC y la comunicación.

Por su parte, la Universidad de Vigo crea seis competencias generales que dan cabida a otras más específicas. Las primeras son eminentemente procedimentales (saber hacer) mientras que en las segundas se recogen, entre otras, aquellas que el sector consideraba más importantes.

La Universidad de Girona entiende las competencias relacionadas con el inglés, las TIC y las técnicas de comunicación como transversales, e incorpora las restantes entre las específicas.

La orientación de servicio al cliente y el manejo de técnicas de comunicación son de las competencias más reiteradas en el plan de la Universidad Rey Juan Carlos. Trabajar en inglés y el manejo de las TIC también están presentes en numerosas materias.

La Universidad de Zaragoza confiere más importancia a la orientación al cliente y al manejo de las TIC y, a diferencia del resto, considera las competencias relacionadas con trabajar en inglés específicas del área de idiomas y no las vincula a otras.

Finalizamos nuestro análisis con la Universidad de Huelva, que tiene un listado de competencias más amplio que el de la mayoría de planes porque, en la elaboración de la Memoria, se pidió a cada una de las áreas que incorporara las competencias que consideraba que los alumnos debían adquirir, lo que dio como resultado un alto número de competencias específicas de las distintas disciplinas. En este plan también vemos que las relacionadas con la orientación de servicio al cliente y las TIC se consideran transversales. Trabajar en inglés como lengua extranjera y manejar técnicas de comunicación también están presentes en un alto número de materias.

En el caso de la competencia "Definir objetivos, estrategias y políticas comerciales (CE11)" aparece, de forma más difusa, en las diferentes Memorias adaptada como competencia específica propia de las Áreas de Organización de Empresas e Comercialización e Investigación de Mercados.

\section{Conclusiones}

En el proceso de desarrollo local, la Universidad debe constituirse en un agente dinamizador de la sociedad, a través de la formación básica, continua y com- 
plementaria. La adaptación de los estudios al entorno económico y social, lejos de ser utilitarismo, debe proporcionar una formación adecuada para la cualificación laboral de sus egresados.

Desde la implantación universitaria de los estudios de Turismo como Diplomatura, se ha venido viendo la proliferación en diferentes Universidades de este estos estudios, de igual manera la implementación de Grados resulta importante, pero debe suponer un giro hacia la profesionalización de los graduados y una mejora sustancial en su inserción laboral.

Las competencias mejor valoradas por el sector tienen peso importante dentro de los nuevos planes de estudio (en muchos casos se consideran, además, transversales). De esta forma, podríamos afirmar que los planes recogen competencias relacionadas con los cambios que experimenta el sector. Si los titulados desarrollan las mismas, entre otras, estarían preparados para hacer frente a dichos cambios.

Aunque el planteamiento de la formación por competencias parece positivo y coherente con las demandas del sector, se propone ampliar la investigación con un sistema de indicadores que teste, desde Universidad y empresa, la adquisición de competencias por parte del alumnado una vez finalizados sus estudios. Una valoración positiva significaría que se ha logrado el objetivo, se han formado a los profesionales adecuados. En el caso contrario, habría que plantear medidas correctoras.

\section{BIBLIOGRAFÍA Y FUENTES DOCUMENTALES}

ALBORNOZ, L. (1999): "Opinión del sector empleador sobre las fortalezas y debilidades del Pasante Técnico en Administración de Empresas Turísticas de la Universidad de Oriente, Núcleo de Nueva Esparta". Tesis de Grado no publicada. Universidad de Oriente, Nueva Esparta (citado por Gómez, 2009).

ALBURQUERQUE, F. (1997): Metodología para el desarrollo económico local, Direcciónde Desarrollo y Gestión Local. Instituto Latinoamericano y del Caribe de Planificación Económica y Social. Naciones Unidas / CEPAL, Consejo Regional de Planificación, Santiago de Chile.

ANECA (2005): Libro Blanco del Grado en Turismo, ANECA, Madrid.

CASANOVA, F. (2004): Desarrollo local, tejidos productivos y formación: abordajes alternativos para la formación y el trabajo de los jóvenes, CINTERFOR, Montevideo, $161 \mathrm{pp}$.

CIENFUEGOS VELASCO, M. A. Y AGUILAR MIRANDA, A. A. P. (2011): "Actores/agentes universitarios para el desarrollo local", en Espacios Públicos, vol. 14, núm. 31, mayo-agosto, 2011, pp. 216-226. Disponible en red: $\underline{\text { http://www.redalyc.org/src/inicio/ArtPdfRed.jsp?iCve=67621192012 }}$ (última consulta: 18/03/2013). 
CRUE (2013): Guía de Titulaciones, Conferencia de Rectores de Universidades Españolas. Documento on-line: http://guiatitulaciones.crue.org/base/ (última consulta: 17/05/2013).

GÓMEZ DE LUNAR, V. (2009): "Desempeño laboral del licenciado en Turismo de la Universidad de Oriente, según la opinión del sector empresarial turístico privado de la Isla de Margarita, Estado Nueva Esparta", en Gestión Turística, 11, pp. 41-68.

GUTIÉRREZ SIVIRA, F. (2006): "Desarrollo local-endógeno y el papel de las Universidades en la formación de cultura emprendedora e innovadora en territorios sociodeprimidos", en Laurus, año/vol. 12, no 022, pp. 139-152.

HERRERA, S. I. (2011): "Desafíos en la Formación Universitaria vinculada al Desarrollo Local", en KAIROS. Revista de Temas Sociales, Año 15. $\mathrm{N}^{\mathrm{o}}$ 28. Disponible en red: http://www.revistakairos.org (última consulta: $18 / 03 / 2013)$.

LUCAS, R. (1988): "On the Mechanics of Economic Development", en Journal of Monetary Economics, 22, pp. 3-42 (citado por Herrera, 2001).

MADOERY, O. (2006). "La formación de agentes de desarrollo desde la perspectiva endógena", en XI Congreso Internacional del CLAD sobre la Reforma del Estado y de la Administración Pública, Ciudad de Guatemala, pp. 7-10.

MÁRQUEZ, J. Y DÍAZ, J. (2005): "Formación del recurso humano por competencias", en Sapiens, vol. 6, 1, pp. 85-105.

POON, A. (1993): Tourism, Technology and Competitive Strategies, Cab Intern, Wallingford.

RODRÍGUEZ GUTIÉRREZ, F. Y VILLENEUVE, R. (2001) "El papel de las universidades en la activación de procesos de desarrollo territorial", en Ería, 56, pp. 294-298.

URIEL, E. Y MONFORT, V. (Dir.) (2001): El sector turístico en España, Caja de Ahorros del Mediterráneo, Alicante. 